\title{
Genotype-phenotype studies in a large cohort of Armenian patients with familial Mediterranean fever suggest clinical disease with heterozygous MEFV mutations
}

\author{
Mike M Moradian $^{1,2}$, Tamara Sarkisian ${ }^{1}$, Hasmik Ajrapetyan ${ }^{1}$ and Nare Avanesian ${ }^{2}$ \\ Familial Mediterranean fever (FMF) is an autoinflammatory disorder generally caused by recessively inherited mutations in the \\ MEFV gene. FMF is quite prevalent in Armenian population in which majority of patients have two mutated alleles, yet in $18 \%$ \\ of symptomatic patients just one mutation has been detected. To explain this finding, we analyzed the symptoms and genotypes \\ of 1299 patients, including 236 affected heterozygous patients with definite diagnosis of FMF. We selected a subset of 63 \\ heterozygous, homozygous and asymptomatic normal individuals and completely sequenced their MEFV genes (exons) to \\ discover any other mutations potentially missed by currently used screening method. Besides four synonymous polymorphisms \\ in exon two and five, we found a T267I mutation in one heterozygous patient with a severe case of FMF who should have been \\ designated as compound heterozygous, yet the other genotypes were all accurate. We used binomial probability distribution \\ of symptoms in homozygous FMF patients to estimate the likelihood of their occurrences in heterozygous patients and \\ demonstrated the assemblage of patients into groups with similar clinical criteria using statistical clustering. We found \\ extremely high probabilities for the presence of FMF symptoms in heterozygous individuals and determined that symptoms were \\ equally likely to occur in both analyzed genotypes. Therefore, our study supports the rising evidence that a single MEFV \\ mutation could be associated with mild FMF symptoms. However, heterozygous patients presenting with severe phenotype \\ should be further analyzed for less common second MEFV mutation using gene sequencing. \\ Journal of Human Genetics (2010) 55, 389-393; doi:10.1038/jhg.2010.52; published online 20 May 2010
}

Keywords: Armenian; FMF; heterozygous; homozygous; MEFV; pathogenicity; symptoms

\section{INTRODUCTION}

Familial Mediterranean fever (FMF) is an autoinflammatory disease characterized by recurrent episodes of fever, peritonitis, pleuritis, synovitis and complications of amyloidosis. ${ }^{1}$ This autosomal recessive condition is usually caused by mutations in both alleles of the MEFV gene. ${ }^{2,3}$ Correlation between genotype and phenotype in individuals affected by the FMF has long been a subject of discussion. Studies from different populations have suggested significant variance in symptoms and pathogenicity of the disease with regard to corresponding mutations. ${ }^{4,5}$ More than 30 mutations in the $M E F V$ gene, which codes for a protein called pyrin, have been attributed to causing FMF with the most prevalent and common mutations in different populations being M694V, V726A, M680I, E148Q and M694I. ${ }^{6}$ The symptoms and severity of the inflammation vary depending on the type and number of mutations in the $M E F V$ gene, which could result in heterozygous, homozygous, compound heterozygous and patients with three or more mutations and complex alleles. A good example of high penetrance is $\mathrm{M} 694 \mathrm{~V}$, which causes the most frequent attacks and amyloidosis. ${ }^{4}$ There are a variety of symptoms in FMF with the most common being the following: fever, abdominal and thoracic pain, joint pain and inflammation, skin eruptions, myalgia (muscle pain) and amyloidosis. Of course, the homozygous and compound heterozygous mutations are highly likely to cause such complications in FMF patients; however, some affected individuals possess just one of the common mutations in the $M E F V$ gene, suggesting that one mutated allele may be able to cause the symptoms. ${ }^{7}$

Several studies have analyzed the symptoms caused by the MEFV mutations and tried to draw conclusions on the frequency of such mutations, their pathogenicity and clinical manifestation. ${ }^{8-10}$ Although we greatly benefit from such studies, they target a small patient cohort, patients with a specific mutation or from a single or small geographical location. The Center of Medical Genetics in the Republic of Armenia has diagnosed well over 5000 FMF patients, a significantly high number relative to the size of the Armenian

${ }^{1}$ Center of Medical Genetics and Primary Health Care, Department of Molecular Genetics, Yerevan, Armenia and ${ }^{2}$ Department of Medical Genetics, Morava Inc., Glendale, CA, USA Correspondence: Dr MM Moradian, Department of Medical \& Molecular Genetics, Morava Inc., 1035 Melrose Avenue \#D, Glendale, CA 91202, USA. E-mail: moradian@makgene.com

Received 4 January 2010; revised 14 April 2010; accepted 19 April 2010; published online 20 May 2010 
population. In this study, we took advantage of a large patient cohort and analyzed a subset of 1299 FMF patients, with sufficient genotype and phenotype information to demonstrate the similarities and differences between the clinical criteria used for FMF diagnosis in homozygous and heterozygous patients. Subsequently, we completely sequenced the $M E F V$ gene from 63 homozygous, heterozygous patients and healthy controls to identify potentially undetected, uncommon or unknown mutations.

\section{MATERIALS AND METHODS}

Clinical diagnosis of FMF was conducted using Tel-Hashomer clinical criteria. ${ }^{11}$ Patient samples were then tested for the 12 most common mutations within the Armenian population. The FMF StripAssay, ${ }^{12,13}$ which is based on the reversehybridization principle, was used to identify the following $12 \mathrm{MEFV}$ mutations: E148Q, P369S, F479L, M680I (G/C), M680I (G/A), I692del, M694V, M694I, K695R, V726A, A744S and R761H. A total of 63 samples, 20 homozygous or compound heterozygous, 23 heterozygous and 20 asymptomatic wild-type controls, were selected and their $M E F V$ gene was completely sequenced independently in the United States using dideoxy termination methodology on an ABI 3100 capillary electrophoresis instrument (Appliedbiosystems Inc., Carlsbad, CA, USA). The sequencing facility and the designed primers for all exons were provided by Morava Inc. (Glendale, CA, USA).

\section{Statistical analysis}

Clustering of data was carried out using symptoms from 345 homozygous patients with a definite diagnosis of FMF and all heterozygous patients with M694V, V726A, M680I, E148Q, F479L and R761H (ordered based on the number of cases) to reduce symptom bias. We chose 11 Tel-Hashomer clinical criteria, which contained sufficient data, to generate informative clustering using the powerful statistical analysis tool MATLAB (The MathWorks, Inc., Natick, MA, USA). We also used binomial probability distribution to accurately estimate the probabilities for occurrence of each clinical criterion in hetero- zygous individuals. The probability $p$ was obtained from homozygous patients with a definite diagnosis of FMF and was used in the following equation:

$$
\text { Probability of }(K \text { out of } N)=\frac{N ! *\left(p^{K}\right)\left(q^{N-K}\right)}{K !(N-K) !}
$$

where $N$ was the number of heterozygous individuals, $K$ was the number of individuals who were positive for a specific clinical criterion, probability $p$ was obtained from definite homozygous cases, and $q=p-1$. Probabilities for all possible values of $K, 0 \leqslant \mathrm{~K} \leqslant \mathrm{~N}$, were calculated and the final probability was obtained by summing all probabilities of $K \ldots N$ to determine the probability of having $K$ or more heterozygous individuals with that particular clinical criterion. Probability $p$ for each criterion was also used to calculate the probability of success in heterozygotes, which in our case noted the presence of that criterion.

\section{RESULTS}

\section{FMF patient statistics}

Initially, we analyzed a total of 1734 individuals with mutated $M E F V$ genes and grouped them into three categories of homozygous, heterozygous and compound heterozygous mutations. The main symptoms of these patients were as follows: fever 1467 (85\%), abdominal pain $1383(81 \%)$, pleuritis $952(55 \%)$, arthralgia 742 (43\%), myalgia $533(31 \%)$, erysipelas-like erythema $282(16 \%)$, splenomegaly $224(13 \%)$ and amyloidosis $32(2 \%)$. The age of onset was below 30 years in $95 \%$ of the patients, with $50 \%$ manifesting the symptoms before the age of 5 years. Also in $27 \%$ of the patients, the frequency of symptoms recurrence was at least twice a month. Details of different symptoms, average age of onset, and average duration and frequency of attacks for all homozygous and heterozygous genotypes analyzed in this study are shown in Table 1 . The average value of the data presented in this table is fairly comparable to a recent study

Table 1 Details of different symptoms, average age of onset, and average duration and frequency of attacks for all homozygous and heterozygous genotypes

\begin{tabular}{|c|c|c|c|c|c|c|c|c|c|c|}
\hline Genotypes & M694V/M694V & M694V/- & V726AN726A & V726A/- & M680I/M680I & M680I/- & $E 148 Q /-$ & F479L/- & $R 761 H / R 761 H$ & $R 761 H_{-}$ \\
\hline Number of patients analyzed & 320 & 207 & 38 & 72 & 46 & 42 & 19 & 6 & 6 & 5 \\
\hline Fever & $285(89)$ & $148(71)$ & 37 (97) & $48(66)$ & $41(89)$ & $31(73)$ & $11(57)$ & $5(83)$ & $6(100)$ & $5(100)$ \\
\hline Abdominal pain & $264(82)$ & $140(67)$ & $31(81)$ & $49(68)$ & $38(82)$ & $32(76)$ & $11(57)$ & $5(83)$ & $4(66)$ & $1(20)$ \\
\hline Arthralgia & $170(53)$ & $79(38)$ & $26(68)$ & $11(15)$ & $27(58)$ & $15(35)$ & $4(21)$ & $2(33)$ & $3(50)$ & $0(0)$ \\
\hline Pleuritis & $196(61)$ & $73(35)$ & $14(36)$ & $24(33)$ & $20(43)$ & $23(54)$ & $4(21)$ & $3(50)$ & $1(16)$ & $0(0)$ \\
\hline Myalgia & $67(21)$ & $48(23)$ & $2(5)$ & $14(19)$ & $7(15)$ & $11(26)$ & $2(10)$ & $2(33)$ & $0(0)$ & $1(20)$ \\
\hline Erysipelas erythema & $117(36)$ & $30(14)$ & $19(50)$ & $7(9)$ & $20(43)$ & $6(14)$ & $2(10)$ & $0(0)$ & $0(0)$ & $0(0)$ \\
\hline Amyloidosis & $10(3)$ & $6(2)$ & $3(7)$ & $2(2)$ & $1(2)$ & $1(2)$ & $0(0)$ & $0(0)$ & $0(0)$ & $0(0)$ \\
\hline Fever-abdominal pain & $246(76)$ & $122(59)$ & $31(81)$ & $39(54)$ & $37(80)$ & $27(64)$ & $11(57)$ & $4(66)$ & $4(66)$ & $1(20)$ \\
\hline Fever-arthralgia & $160(49)$ & $70(33)$ & $25(65)$ & $10(13)$ & $26(56)$ & $13(31)$ & $4(21)$ & $2(33)$ & $3(50)$ & $0(0)$ \\
\hline Fever-pleuritis & $185(57)$ & $65(31)$ & $13(34)$ & $18(25)$ & $20(43)$ & $20(47)$ & $4(21)$ & $3(50)$ & $1(16)$ & $0(0)$ \\
\hline Fever-myalgia & $109(34)$ & $43(20)$ & $19(50)$ & $11(15)$ & $18(39)$ & $8(19)$ & $1(5)$ & $2(33)$ & $0(0)$ & $0(0)$ \\
\hline Fever-abdominal pain-pleuritis & $142(44)$ & $59(28)$ & $20(52)$ & $17(23)$ & $23(50)$ & $17(40)$ & $3(15)$ & $3(50)$ & $2(33)$ & $0(0)$ \\
\hline Fever-abdominal pain-arthralgia & $77(24)$ & $59(28)$ & $11(28)$ & $16(22)$ & 17 (37) & $10(23)$ & $2(10)$ & $1(16)$ & $1(16)$ & $0(0)$ \\
\hline fever-abdominal pain-myalgia & $97(30)$ & $41(19)$ & $16(42)$ & $10(13)$ & $17(37)$ & $7(16)$ & $0(0)$ & $2(33)$ & $0(0)$ & $1(20)$ \\
\hline Fever-pleuritis-myalgia & $66(20)$ & $31(15)$ & $11(28)$ & $8(11)$ & $12(26)$ & $7(16)$ & $0(0)$ & $1(16)$ & $0(0)$ & $0(0)$ \\
\hline Fever-arthralgia-myalgia & $75(23)$ & $28(13)$ & 7 (18) & $6(8)$ & $10(21)$ & $4(9)$ & $0(0)$ & $0(0)$ & $0(0)$ & $0(0)$ \\
\hline $\begin{array}{l}\text { Fever-abdominal } \\
\text { pain-pleuritis-arthralgia-myalgia }\end{array}$ & $46(14)$ & $19(9)$ & $4(10)$ & $3(4)$ & $7(15)$ & $3(7)$ & $0(0)$ & $0(0)$ & $0(0)$ & $0(0)$ \\
\hline Average age of onset & 5.1 & 10.9 & 10.2 & 9.4 & 9.8 & 9.9 & 18.7 & 13.5 & NA & NA \\
\hline Average duration of attacks in days & 3.6 & 2.6 & 2.5 & 2.7 & 4.0 & 2.8 & 1.9 & 3.1 & NA & NA \\
\hline Average frequency of attacks & $1-2$ weeks & 2-3 weeks & 2-3 weeks & 3-4 weeks & 2-3 weeks & $2-3$ weeks & 1 month & 1 month & NA & NA \\
\hline
\end{tabular}

Abbreviation: NA, not applicable.

Symptoms are presented by the number of affected patients and their percentages in parenthesis. 
conducted by Booty et al., ${ }^{14}$ yet we found a few differences when we compared our detailed heterozygous patients' results with those of a relatively larger study ( 94 heterozygous patients) conducted by Kone-Paut et al. ${ }^{15}$ The median age of onset for our heterozygous patients was 7 years ( 1 month-44 years), which was 5 years older than that in their study, yet the range was quite similar. All symptoms occurred with proportional percentages, except pleuritis, which was $50 \%$ higher in our patients. Duration of the attacks were slightly (that is, $20 \%$ ) shorter in our patients and the frequency of attacks in more than half of our patients was $<1$ month, a $37 \%$ difference. Basically, our heterozygous patients had slightly shorter duration of attacks with higher frequencies.

We identified homozygous genotypes for M694V, V726A, M680I, $\mathrm{R} 761 \mathrm{H}$ and one F479L (data not included), and heterozygous genotypes for M694V, V726A, M680I, E148Q, R761H, F479L, and one P369S and K695R (data not included). It was interesting that mutation E148Q was not present in any homozygous form, yet was present in more than $5 \%$ of symptomatic heterozygous and $8.5 \%$ of symptomatic compound heterozygous patients both causing mild to moderate FMF. As per our knowledge, no severe FMF case has been attributed to heterozygous or compound heterozygous genotypes with an E148Q-mutated allele in the Armenian population. Comparison of information in Table 1 indicates correlation of more than $90 \%$ between homozygous and heterozygous data, yet milder symptoms and shorter and less frequent attacks occur in heterozygous patients.

The overall frequency of MEFV mutations in the patients was M694V (56.1\%), V726A (17.9\%), M680I (17.9\%), R761H (3.3\%), E148Q (2.0\%), F479L (1.5\%), and P369S, M694I and R42W with 0.5\% each, which was quite comparable to a previous study conducted by Sarkisian et al. ${ }^{7}$ To eliminate ambiguities, we narrowed our analysis to 1299 patients with a definite diagnosis of FMF (Table 2) in which 345 (27\%) were homozygous, 718 (55\%) were compound heterozygous and the remaining 236 (18\%) were heterozygous genotypes. Table 2 shows the results of genotypes grouped according to the clinical probability and severity score of FMF using Tel-Hashomer clinical criteria. ${ }^{11}$ Not surprisingly, the overwhelming majority of patients with two mutations were either in definite or possible categories, yet surprisingly, $87 \%$ of heterozygous patients fell in those categories with comparable symptoms. Hence, we conducted a severity score study to estimate the severity of the disease in all genotypes (Table 2). In homozygous and compound heterozygous patients, 87 and $71 \%$ suffered from severe or moderate FMF, respectively, in which the picture for heterozygous patients was somewhat different. Almost all (98\%) heterozygous patients belonged to moderate or mild FMF categories, which indicated that some mutations could cause a milder

Table 2 FMF diagnosis and severity classifications of genotypes (Tel-Hashomer criteria)

\begin{tabular}{lcccr}
\hline & Definite & Possible & Unlikely & Total \\
\hline Homozygous & $345(84 \%)$ & $61(15 \%)$ & $4(1 \%)$ & 410 \\
Heterozygous & $236(67 \%)$ & $70(20 \%)$ & $45(13 \%)$ & 351 \\
Compound heterozygous & $718(74 \%)$ & $178(18 \%)$ & $77(8 \%)$ & 973 \\
& & & & \\
& Severe & Moderate & Mild & Total \\
\hline Homozygous & $85(21 \%)$ & $271(67 \%)$ & $54(13 \%)$ & 410 \\
Heterozygous & $5(2 \%)$ & $173(49 \%)$ & $173(49 \%)$ & 351 \\
Compound heterozygous & $60(6 \%)$ & $633(65 \%)$ & $280(29 \%)$ & 973 \\
\hline
\end{tabular}

version of FMF with just one mutated allele. However, surprisingly, five heterozygous patients, two M694V, two V726A and one M680I, turned out to belong to the severe FMF category. Sequencing data (discussed below) showed that at least one of these patients was a compound heterozygous, which suggests that heterozygous patients are highly unlikely to suffer from severe FMF cases. If they do, however, their $M E F V$ gene most probably contains more than one mutation, which could be detected if more sensitive and comprehensive laboratory techniques, such as sequencing, are used.

\section{Statistical analysis}

Results for each genotype and its Tel-Hashomer clinical criteria for definite homozygous and all heterozygous patients are shown in a clustergram in Figure 1. We chose to use a clustergram, which is used to show the differences in assemblage, instead of a regular clinical criteria table because of our enormous data size. We used 11 of the most common clinical criteria to generate this clustergram in which the top part of the graph contains data from 345 definite homozygous patients, excluding symptoms of ambiguous diagnosis, and the bottom part includes all heterozygotes (that is, 351, details in Table 2) to minimize the symptoms bias. We used this clustergram to indicate the presence (yellow lines) or absence (green lines) of the criteria. None of the criteria generated a distinct cluster between homozygous and heterozygous data, which suggests that these analyzed clinical criteria are almost equally likely to occur in both data sets. We completed our statistical analysis by using binomial probability distribution to determine how likely the criteria present in definite homozygous patients are to occur in any heterozygous patient (for calculation details refer to Material and methods). Almost all the clinical criteria were highly likely to occur in heterozygous patients with a probability of $\geqslant 95 \%$, with the exception of abdominal pain, which was $83 \%$ (Figure 1). Although abdominal pain is the most common symptom of FMF, it could also be caused by several other disorders or conditions, and according to our analysis, may not be a significant indicator for symptom correlation between homozygous and heterozygous FMF patients. The criterion for amyloidosis was also not as informative, as it occurs only in severe cases and in very few patients. It was therefore included solely for comparison purposes. High probabilities for the presence of symptoms and clinical criteria in heterozygous patients supported the diagnosis of mild FMF cases in some heterozygous patients.

Figure 2 shows the differences between the severity scores of all 410 homozygous and 351 heterozygous patients. According to Tel-Hashomer criteria, scores of $>10$ suggest severe FMF cases, in which scores between 5 and 10 represent moderate, and 2-5 refer to mild cases. ${ }^{11}$ Figure 2 indicates that we rarely observed heterozygous patients with severe cases or homozygous patients with scores of $<2$. The histogram for homozygous patients was concentrated on moderate to severe regions, where, in contrast, it shifted to moderate to mild regions in heterozygous patients (Figure 2). Analysis of severity scores assisted us in distinguishing and demonstrating that heterozygous patients could be suffering from milder versions of FMF.

\section{Sequencing results}

We completely sequenced all exons in the MEFV genes from 20 homozygous (10 homozygous and 10 compound heterozygous patients), 23 heterozygous patients and 20 normal asymptomatic individuals. Heterozygous patients were chosen according to the latest MEFV mutation frequencies, thus $12 \mathrm{M} 694 \mathrm{~V}, 4 \mathrm{M} 680 \mathrm{I}$ and 4 V726A heterozygous patients were completely sequenced. We also sequenced three patients (one each) with R761H, E148Q and F479L mutations, 


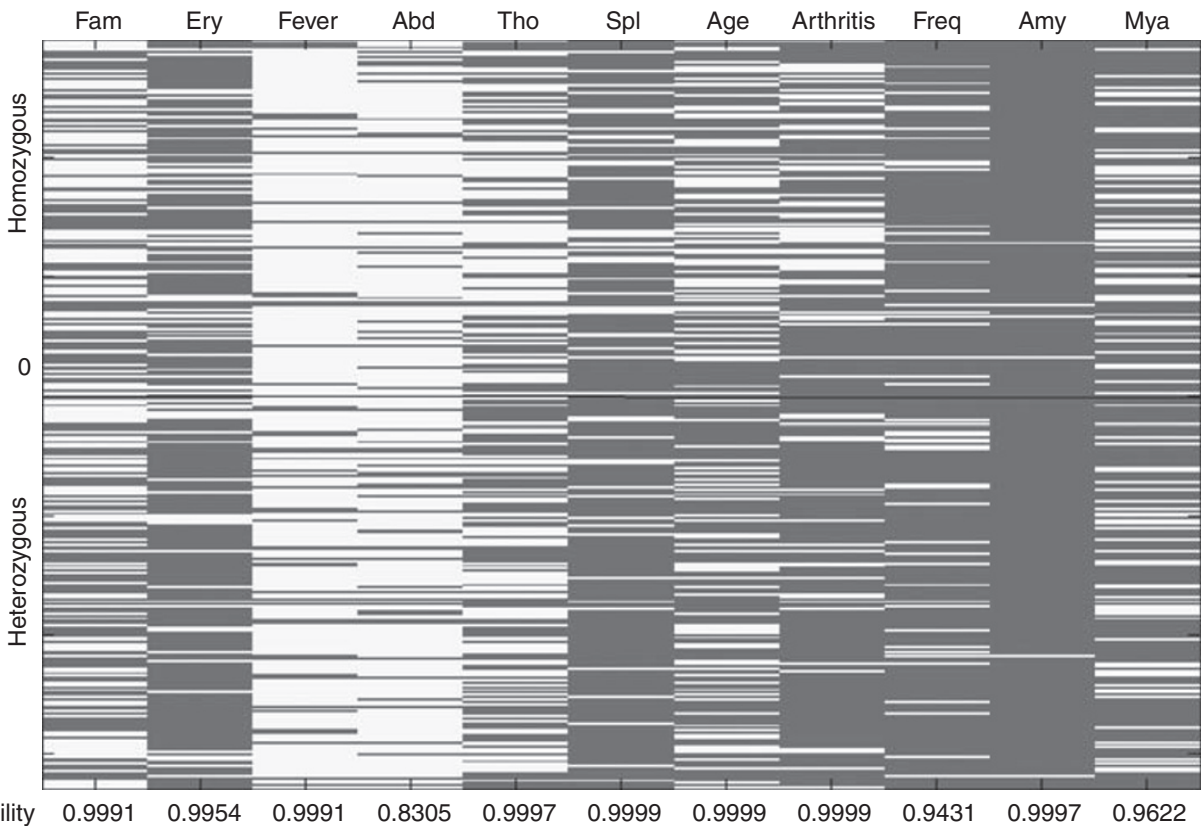

Figure 1 Clinical data of homozygous patients with definite diagnosis of FMF and all heterozygous patients, according to the Tel-Hashomer criteria. Homozygous data come from 345 patients with definite diagnosis of FMF, yet heterozygous data are from all 351 heterozygous patients regardless of their diagnosis status, which includes 236 with definite, 70 with possible, and 45 with unlikely diagnosis of FMF. Fam: family history; Ery: erysipelas-like signs; Abd: abdominal signs; Tho: thoracic signs; Spl: splenomegaly; Age: diagnosed $<5$ years of age; Art: arthralgia, Freq: 2 or more attacks per month; Amy: amyloidosis; Mya: myalgia. Point 0 separated homozygous and heterozygous patients. Yellow (light) lines indicate presence and green (dark) lines indicate absence of the criteria in a given patient. A full color version of this figure is available at the Journal of Human Genetics journal online.

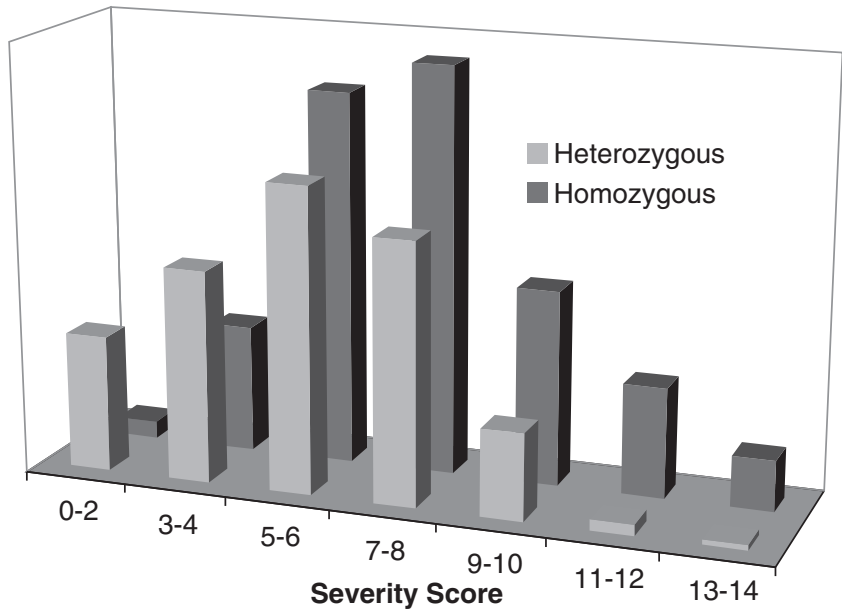

Figure 2 Severity Score histogram of homozygous vs. heterozygous patients. Severity scores on the $\mathrm{X}$-axis are presented in multiples of 2. Y-axis represents the number of patients in each bar.

despite the fact that they did not result in any severe FMF cases. We found synonymous polymorphisms in exons 2 and 5 of seven individuals, which, not surprisingly, could not have contributed to any FMF symptoms. The positions of the polymorphisms from exon 2 were at glycine 138 (GGA to GGG) and alanine 165 (GCC to GCA). In exon 5, these polymorphisms were at glutamic acid position 473 (GAA to GAG) and aspartic acid position 510 (GAT to GAC). Three of these polymorphisms were transitions, yet the substitution at alanine 165 was a transversion, despite the fact that all of them were synonymous. As in synonymous substitutions transversions are less likely to occur than transitions, then suggesting a more variable position for this particular nucleotide substitution $(\mathrm{C} \rightarrow \mathrm{A})$ could be a plausible explanation.

We verified all previously identified mutations using the screening method (described in Materials and method) through sequencing the $M E F V$ gene in homozygous, compound heterozygous, and all but one heterozygous patient. This heterozygous patient previously was determined to have a V726A mutation with a severe case of FMF and sequencing revealed that it also had a T267I undetected mutation resulting in a compound heterozygous genotype. There were five such heterozygous patients with severe cases of FMF and all were candidates for sequencing yet only the patient's sample contained viable DNA, which could be amplified and sequenced. The other four samples were not preserved well and all attempts to obtain new samples were unsuccessful as many patients travel from rural areas to Yerevan infrequently for medical care and, consequently, there were no means of communication with them.

As we were able to identify an undetected mutation in one heterozygous patient with severe FMF, we can perhaps assume that the other four patients were highly likely to have a second undetected mutation in their $M E F V$ gene. If this was the case, then the percentage of undetected mutations in heterozygous patients could be as high as 5 out of 23 or almost $22 \%$. On the other hand, sequencing confirmed that the remaining sequenced heterozygous patients with mild to moderate FMF cases had only one mutation in their $M E F V$ gene, which suggests that mutations in Armenian population, particularly the three most prevalent ones (that is, M694V, M680I and V726A), could cause mild FMF cases with a single mutated allele.

\section{DISCUSSION}

Presence of FMF symptoms in heterozygous patients is often attributed to the inability of screening methods to identify all potentially pathogenic mutations. Therefore, assessing the accuracy of this widely 
used method in the Armenian population became a necessity. Sequencing of all MEFV exons of a subset of our patients verified the accuracy of our screening method, yet indicated that just a small percentage of heterozygous patients suffering from severe FMF cases could be false negatives. Recent studies have also indicated that in just a small percentage of heterozygous individuals, a second mutation in MEFV exons, introns or promoter region has been found. ${ }^{14,15}$ Moreover, a few studies have shown that mutations from other autoinflammatory disorders, as well as MEFV expression levels and copy-number variation, similarly may not be a major contributing factor for FMF symptoms in heterozygous patients. ${ }^{16,17,18}$ Findings from these studies along with our results support the notion that some MEFV mutations may act as a dominantly inherited trait and cause mild FMF cases. If this is the case, then such dominant effect may be due to a single functional allele not producing sufficient gene product for proper function resulting in haploinsufficiency, or some mutations in the $M E F V$ gene altering the pyrin protein product such that it acts as a proinflammatory molecule, a model of gain of function. In addition, as FMF heterozygous genotypes produce a phenotype intermediate between the two homozygotes, it could be said that one allele shows incomplete dominance over the other. Conversely, most of the loss of function mutations in the $M E F V$ gene are recessively inherited and support the hypothesis that pyrin functions as an inhibitor of inflammation. Nonetheless, we suggest that if any heterozygous patient is screened for most common MEFV mutations in their respective population and their symptoms suggest that they are likely to have mild to moderate FMF, then colchicine therapy may be initiated. On the other hand, heterozygous patients who are determined to have a severe case of FMF are highly likely to possess an undetected less common mutation in their $M E F V$ gene and their genotype should be analyzed further through sequencing.

Our statistical analysis indicated high probabilities for the presence of symptoms, common to homozygous FMF cases, in heterozygous patients. These symptoms were less severe and generally caused mild disease, which prompted us to assume that the MEFV gene threshold or its protein product threshold may offer an explanation. Different levels of $M E F V$ gene product may be required to fight inflammation in different parts of the body, depending on the severity of inflammation. In homozygous or compound heterozygous cases, the $M E F V$ gene expresses protein that may not be completely functional, resulting in FMF symptoms. On the other hand, in heterozygous patients one allele produces functional protein, yet the mutated allele may either produce a nonfunctional protein or a partially functional one, depending on the mutation. Therefore, some MEFV mutations could result in the expression of nonfunctional proteins, which in heterozygous patients may result in insufficient protein levels to fight inflammation manifesting mild FMF symptoms. Of course, this issue requires future research to demonstrate how each mutation changes the structure of the MEFV protein product, namely, pyrin. In addition, other physiological, environmental or unknown conditions, which could cause inflammation, may affect the protein threshold or the levels of pyrin required to fight the inflammation.

In this study, we focused on major points that not only supported our hypothesis but also laid the groundwork for more comprehensive studies in future. We analyzed symptoms and genotypes from a large patient cohort (that is, sample size of 1299), which minimized the random error and generated statistically significant analysis. Correla- tion of symptoms between homozygous and heterozygous patients was carried out using unconventional statistical methods used in Bioinformatics to analyze large data sets that rendered more confidence to our analysis. Sequencing of the entire $M E F V$ gene allowed us to detect all potential mutations and to accept our hypothesis that at least three or more of the most prevalent MEFV mutations in the Armenian population could cause mild FMF cases if present in just one allele. Meanwhile, we remain convinced that future comprehensive studies could assist in determining other unknown factors or conditions that may result in the occurrence of FMF or FMF-like symptoms in heterozygous patients of Armenian descent.

\section{ACKNOWLEDGEMENTS}

We thank Dr Matteo Pellegrini for his constructive comments on the statistical analysis of our data.

1 Sohar, E., Gafni, J., Pras, M. \& Heller, H. Familial Mediterranean fever. A survey of 470 cases and review of the literature. Ann. J. Med. 43, 227-253 (1967).

2 International FMF Consortium. Ancient missense mutations in a new member of the RoRet gene family are likely to cause familial Mediterranean fever. Cell 90, 797-807 (1997).

3 French FMF Consortium. A candidate gene for familial Mediterranean fever. Nat. Genet. 17, 25-31 (1997)

4 Cazeneuve, C., Sarkisian, T., Pêcheux, C., Dervichian, M., Nédelec, B., Reinert, P. et al. The MEFV-gene analysis in Armenian patients with familial Mediterranean fever, diagnostic value and unfavorable renal prognosis of the M694V homozygous genotype-genetic and therapeutic implications. Am. J. Hum. Genet. 65, 88-97 (1999).

5 Aksentijevich, I., Torosyan, Y., Samuels, J., Centola, M., Pras, E., Chae, J. J. et al. Mutations and haplotype studies of familial Mediterranean fever reveal new ancestral relationships and evidence for a high carrier frequency with reduced penetrance in the Ashkenazi Jewish population. Am. J. Hum. Genet. 64, 949-962 (1999).

6 Touitou, I. The spectrum of familial Mediterranean fever (FMF) mutations. Eur. J. Hum. Genet. 9, 473-483 (2001).

7 Sarkisian, T., Ayrapetyan, H. \& Shahsuvaryan, G. Molecular study of FMF patients in Armenia. Curr. Drug Targets Inflamm. Allergy 4, 113-116 (2005).

8 Tunca, M., Akar, S., Onen, F., Ozdogan, H., Kasapcopur, O. et al. Familial Mediterranean fever (FMF) in Turkey: results of a nationwide multicenter study. Medicine (Baltimore) 84, 1-11 (2005).

9 Topaloglu, R., Ozaltin, F., Yilmaz, E., Ozen, S., Balci, Y., Besbas, N. et al. E148Q is a disease-causing MEFV mutation: a phenotypic evaluation in patients with familial Mediterranean fever. Ann. Rheum. Dis. 64, 750-752 (2005).

10 Cazeneuve, C., Hovannesyan, Z., Geneviève, D., Hayrapetyan, H., Papin, S., GirodonBoulandet, E. et al. Familial Mediterranean fever among patients from Karabakh and the diagnostic value of the MEFV gene analysis in all classically affected populations. Arthritis Rheum. 48, 2324-2331 (2003).

11 Pras, M. Familial Mediterranean fever, from the clinical syndrome to the cloning of the pyrin gene. Scand. J. Rheum. 27, 92-97 (1998).

12 Bernot, A., Da Silva, C., Petit, J. L., Cruaud, C., Caloustian, C., Castet, V. et al. Nonfounder mutations in the MEFV gene establish this gene as the cause of familial Mediterranean fever (FMF). Hum. Mol. Genet. 7, 1317-1325 (1998).

13 Teletar, M. \& Grody, W. W. Molecular genetic testing for familial Mediterranean fever. Mol. Genet. Metab. 71, 256-260 (2000).

14 Booty, M. G., Chae, J. J., Masters, S. L., Remmers, E. F., Barham, B., Le, J. M. et al. Familial Mediterranean fever with a single MEFV mutation: where is the second hit? Arthritis Rheum. 60, 1575-1577 (2009).

15 Koné-Paut, I., Hentgen, V., Guillaume-Czitrom, S., Compeyrot-Lacassagne, S., Tran, T. A. \& Touitou, I. The clinical spectrum of 94 patients carrying a single mutated MEFV allele. Rheumatology 48, 840-842 (2009).

16 Marek-Yagel, D., Berkun, Y., Padeh, S., Abu, A., Reznik-Wolf, H., Livneh, L. et al. Clinical disease among patients heterozygous for familial Mediterranean fever. Arthritis Rheum. 60, 1575-1577 (2009).

17 Notarnicola, C., Boizet-Bonhoure, B., De Santa Barbara, P., Osta, M. A., Cattan, D. \& Touitou, I. Characterization of new mutations in the $5^{\prime}$-flanking region of the familial Mediterranean fever gene. Genes Immun. 10, 273-279 (2009).

18 van Gijn, M. E., Soler, S., de la Chapelle, C., Mulder, M., Ritorre, C., Kriek, M. et al. Search for copy number alterations in the MEFV gene using multiplex ligation probe amplification, experience from three diagnostic centres. Eur. J. Hum. Genet. 16, 1404-1406 (2008). 